\title{
Statistical model of the powder flow regulation by nanomaterials
}

\author{
D. Kurfeß ${ }^{\text {a,* }}$, H. Hinrichsen ${ }^{\text {a }}$, I. Zimmermann ${ }^{\text {b }}$ \\ ${ }^{a}$ Fakultät für Physik und Astronomie, Universität Würzburg, Am Hubland, \\ D-97074 Würzburg, Germany \\ ${ }^{\mathrm{b}}$ Fakultät für Chemie und Pharmazie, Universität Würzburg, Am Hubland, \\ D-97074 Würzburg, Germany
}

\begin{abstract}
Fine powders often tend to agglomerate due to van der Waals forces between the particles. These forces can be reduced significantly by covering the particles with nanoscaled adsorbates, as shown by recent experiments. In the present work a quantitative statistical analysis of the effect of powder flow regulating nanomaterials on the adhesive forces in powders is given. Covering two spherical powder particles randomly with nanoadsorbates we compute the decrease of the mutual van der Waals force. The dependence of the force on the relative surface coverage obeys a scaling form which is independent of the used materials. The predictions by our simulations are compared to the experimental results.
\end{abstract}

Key words: Agglomeration, Aggregation, Glidants, Granular flow, Pharmaceuticals, Powders

PACS: 45.70.-n, 82.60.Qr, 83.10.Rs

\section{Introduction}

Dry granular powders are basic materials of great importance to the pharmaceutical industry. Bulk powders tend to agglomerate due to adhesive forces between the powder particles. This characteristic causes problems in the manufacturing process of drugs, where accurate dosing is essential [1]. Furthermore, insufficient flowability can severely harm production devices as well as prevent inhalable drugs from reaching the lung's alveoli.

\footnotetext{
* Corresponding author.

Email address: physik@kurfess.net (D. Kurfeß).
} 

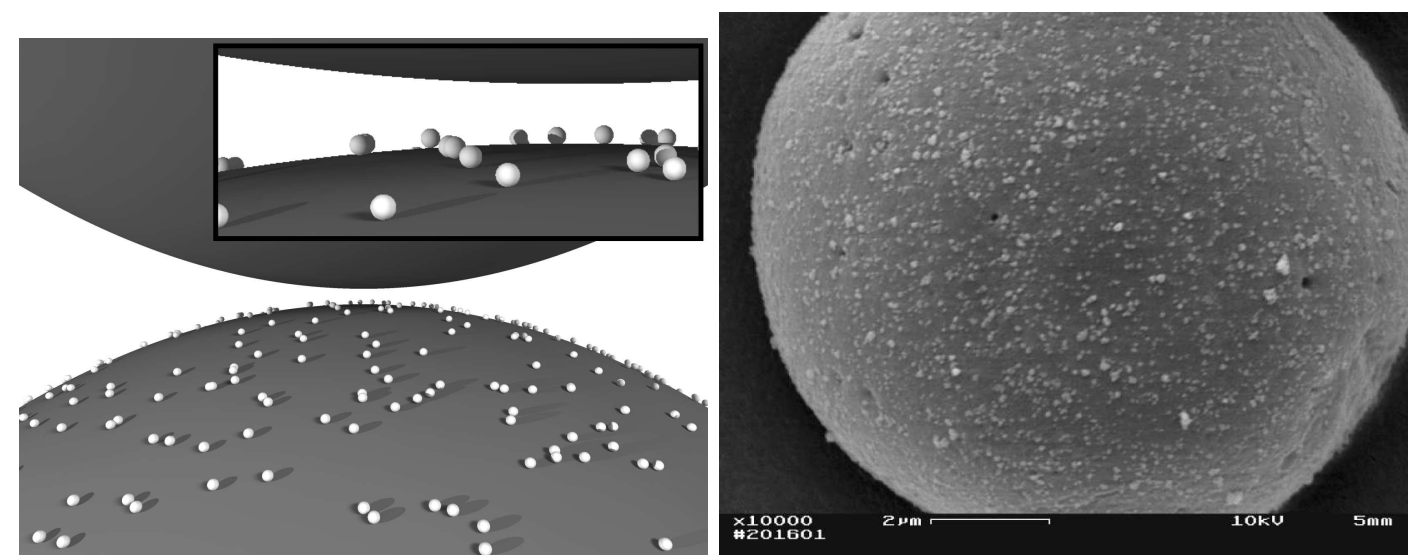

Fig. 1. (left) Model of a covered powder particle of radius $R=11 \mu \mathrm{m}$ and nanoadsorbates of $r=50 \mathrm{~nm}$ (relative surface coverage of $2 \%$ on the lower sphere). Above, a second uncovered particle is approaching. The inset displays a zoomed view of the contact field. (right) Microscopic photo of a corn starch particle covered with aggregates of the nanomaterial Aerosil ${ }^{\circledR} 300$, taken from [2].

Pharmaceutical powders typically have particle diameters on the micrometer scale and contact distances between the particles on the angstrom scale. If they are dry and not highly electrified, van der Waals forces are the strongest interparticle forces, exceeding gravitational and Coulomb forces significantly. As van der Waals forces are short-range it is possible to effectively decrease these adhesive forces by increasing the surface roughnesses of the powder particles by covering them with nanoparticles (cf. Fig. 1). This was verified by tensile strength experiments, in which two powder layers were separated and the required force was measured $[2,3]$.

H.C. Hamaker calculated the van der Waals forces between macroscopic spherical bodies by integration over all molecular dipole-dipole interactions [4]. Accordingly

$$
F_{\mathrm{vdW}}=-\frac{A}{6 H^{2}} \frac{R_{1} R_{2}}{R_{1}+R_{2}}
$$

is the attractive interparticle force between two spheres of radii $R_{1}$ and $R_{2}$, where all material constants are combined into the so called Hamaker constant $A$. $H$ is the distance between the surfaces of the two bodies. In order to avoid a diverging force upon contact, one usually assumes that $H$ cannot become smaller than a so called contact distance $D=4 \times 10^{-10} \mathrm{~m}[5,6,3]$.

K. Meyer theoretically described the decrease of the tensile strength with increasing surface coverage assuming a stable three-point contact, i.e., effectively three nanoparticles act as spacers between two powder particles and combine them [2]. In bulk powders, two particles firstly joined by a one- or two-point contact are subject to torque, and their bonding remains unstable until a 
three- (or more-)point contact is reached.

Meyer considered an idealized system in which the three contact adsorbates are fixed on the vertices of an equilateral triangle $[2,3]$. This model allows one to calculate analytically the distance of the two bulk powder particles against the side length of this triangle. From this distance the interparticle force can immediately be derived using Hamaker's sphere-sphere model.

The result of this calculation describes only two states sharply separated by a critical surface coverage $\rho_{c}$. For $\rho<\rho_{c}$ the powder particles touch and attract each other strongly, while otherwise the nanomaterial establishes a gap between them and the short-range adhesive force vanishes nearly entirely. Obviously Meyer's model of equidistant spacing of the nanoparticles ignores any stochastic aspects which are relevant in reality.

It is the aim of this paper to give a more realistic theoretical description of the effect of flow regulating nanomaterials on the interparticle forces acting between the larger powder particles. We simulate random coverages of the surfaces of the host particles with nanoparticles and calculate the van der Waals forces averaged over many realizations of randomness. We use spherical host particles and nanoadsorbates as a first approximation of reality, as suggested by microscopic photos of corn starch powder particles mixed with nanomaterials, see Fig. 1. Our generated data indicates a scale invariant behavior, where an incomplete gamma function fits the resulting scaling function perfectly. Our results are compared to experimental data, demonstrating the improvements compared to Meyer's model.

\section{Simulation}

The simulation computes the averaged van der Waals force $F_{\mathrm{vdW}}$ versus the relative surface coverage $\rho$. The algorithm consists of three parts: (i) the preparation of a host particle by covering it randomly with adsorbates, (ii) the positioning of two host particles so that a stable three-point contact is formed, and (iii) the computation of the van der Waals force by summation over all resulting sphere-sphere interactions according to the Hamaker model. So each run of this algorithm gives one sample of the adhesive force which can occur.

In the following the three steps of the simulation are shortly explained (for more detailed information see Appendix A). 

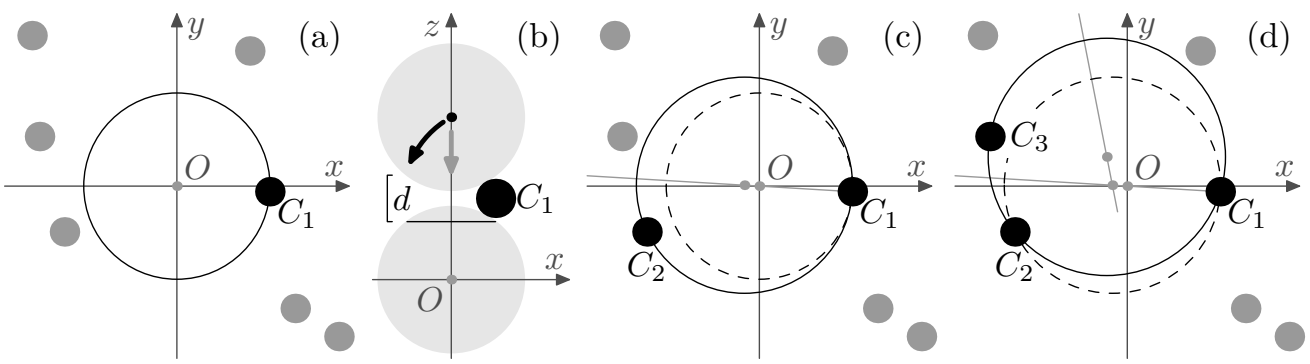

Fig. 2. Algorithm for finding the three contact points. (a) Top view on the covered host particle (white background) and (b) side view. The large dots in (a) are randomly distributed adsorbate particles. The uncovered powder (host) particle is approaching from above along the $z$-axis. The first adsorbate to be hit by the approaching sphere is the one which is located nearest to the $z$-axis $\left(C_{1}\right)$. Upon contact the circle in (a) describes the line of equal distance $d$ in $z$-direction between the two host particles. $C_{1}$ withstands the approaching sphere, so that the momentum of this moving sphere results in a torque (black arrow). (c) Due to the torque the upper host particle rolls off so that its center projected onto the $x-y$-plane moves along $\overline{C_{1} O}$, until this host sphere hits the second contact adsorbate $\left(C_{2}\right)$. (d) Now $C_{1}$ as well as $C_{2}$ hold the approaching host sphere so that its center (projected onto the $x$-y-plane) moves along the perpendicular bisector of the side $C_{1} C_{2}$, until a stable three-point contact is formed.

\subsection{Preparation}

Initially, a host sphere is randomly and successively covered with nanoparticles, so that a uniform (Poisson) distribution with respect to the spherical surface area is established. Unlike the random sequential adsorption (RSA) model, our model does allow the adsorbate particles to overlap. Yet for the low coverages which we are interested in ( $\rho$ has the order of magnitude of few percent), the probability of two overlapping nanoparticles is negligible.

\subsection{Positioning}

In the second part of the algorithm a covered host and (for simplicity reasons) an uncovered powder sphere are brought to collide, cf. Fig. 1 left. The uncovered large powder sphere rolls off over the small adsorbates, until a stable three-point contact builds up (see Fig. 2); or, if the three potential contact candidates are too far away from each other, it rolls off until the two powder spheres directly touch each other. Knowing the characteristics of the contact, it is easy to calculate the new position $\vec{s}$ of the center of the uncovered powder sphere relative to the center of the covered host sphere, which is assumed to be located at the origin. 


\subsection{Computation of the force}

The coordinates $\vec{a}_{i}$ describe the center positions of the $N_{A}$ adsorbates on the covered host particle. Let $R$ be the radius of the powder particles, and $r$ the common radius of all nanoadsorbates. $A_{H H}$ and $A_{A H}$ are the host-host and the adsorbate-host Hamaker constants, respectively. By vectorial summation over all sphere-sphere interactions according to Eq. (1) with two (host) powder particles, we get

$$
\vec{F}_{\mathrm{vdW}}=-\frac{A_{H H}}{6(|\vec{s}|-2 R)^{2}} \frac{R}{2} \cdot \frac{\vec{s}}{|\vec{s}|}-\sum_{i=1}^{N_{A}} \frac{A_{A H}}{6\left(\left|\vec{s}-\vec{a}_{i}\right|-r-R\right)^{2}} \cdot \frac{\vec{s}-\vec{a}_{i}}{\left|\vec{s}-\vec{a}_{i}\right|}
$$

as the resulting interparticle force.

Note that not only the three contact adsorbates but also all other adsorbate particles without direct contact to the uncovered powder sphere are taken into account. Most of them give only minor contributions to the adhesive force, as they are far away from the three-point contact and are therefore distant from the uncovered powder sphere. However, statistically some of them may come very close to the three-point contact and give force contributions of significant order of magnitude. Thus for a complete treatment of randomness it is reasonable to consider the influence of all the adsorbate particles for the computation of the force.

\section{Results and discussion}

For the further discussion, $A=A_{H H}=A_{A H}$ is assumed; most condensed phases, solid as well as liquid ones, have Hamaker constants of about $10^{-19} \mathrm{~J}$ interacting across vacuum, and similar values in air $[7,6,3]$.

Concerning the adhesive effect between two particles, we have to examine only the absolute value of the van der Waals force, $F=\left\langle\left|\vec{F}_{\mathrm{vdW}}\right|\right\rangle$ as the mean of many samples generated by our algorithm. Some results of our simulations are shown in Fig. 3 (for details about the comparison with Meyer's model cf. Appendix B). 


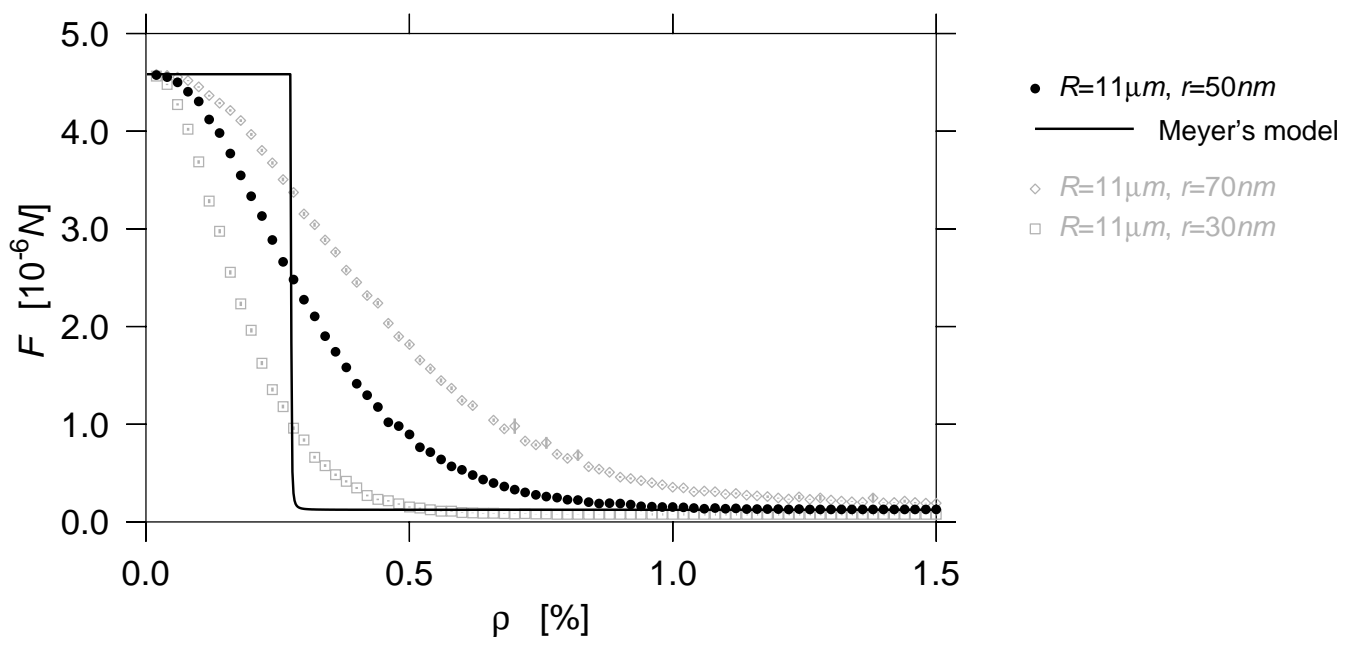

Fig. 3. Simulated data of the van der Waals force versus the relative surface coverage ( $A=8 \times 10^{-19} \mathrm{~J}$, mean of $10^{4}$ samples per point and standard error plotted). For comparison Meyer's model for $R=11 \mu \mathrm{m}$ and $r=50 \mathrm{~nm}$ is displayed.

\subsection{Scaling}

\subsubsection{Scaling properties}

For $\rho \rightarrow 0$, the force approaches the adhesion between two uncovered powder spheres,

$$
F \rightarrow F_{0}=\frac{A \cdot R}{12 D^{2}} \propto R
$$

In Fig. 3 a linear right shift of the decrease of $F$ in dependence of the surface coverage $\rho$ for increasing $r$ can be observed. This suggests the scaling form

$$
\frac{F}{R} \approx f\left(\frac{\rho R}{r}\right)
$$

The collapse of the data in Fig. 4 (top) shows that this scaling form works well for small values of $\rho R / r$, while for small $F / R$ the curves become constant at different values, which grow roughly $\propto r / R$. This seems plausible, because with a large gap between the two host particles the force is mostly affected by the three contact adsorbates only:

$$
\min (F) \approx F_{m}=3 \cdot \frac{A}{6 D^{2}} \frac{R r}{R+r} \propto r
$$

for $r \ll R$. 


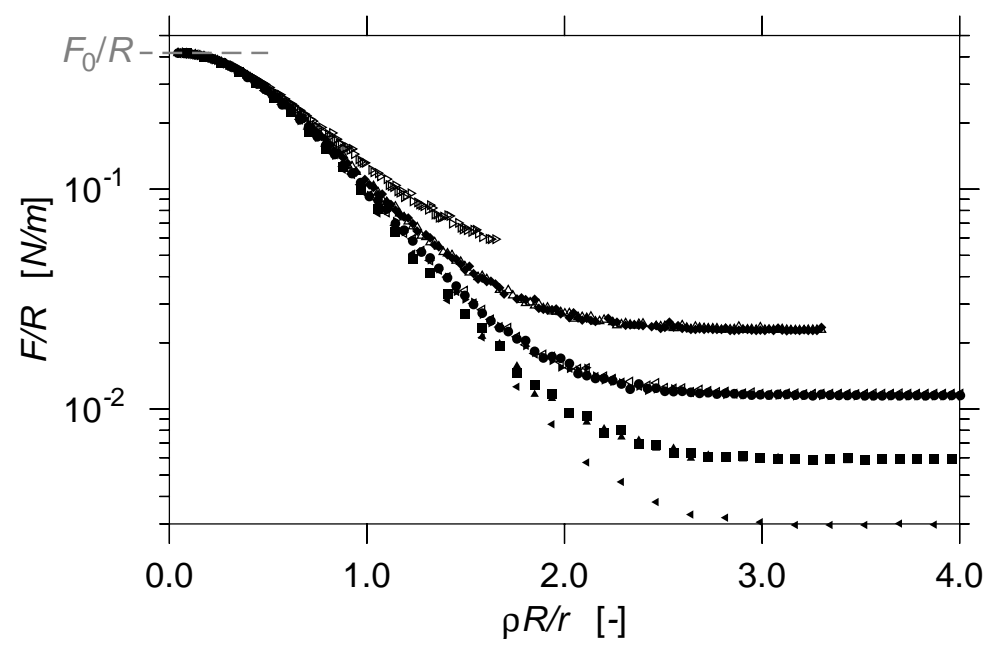

$\triangleleft R=5.5 \times 10^{-6} \mathrm{~m}, r=2.5 \times 10^{-8} \mathrm{~m}$

$\triangle R=5.5 \times 10^{-6} \mathrm{~m}, r=5.0 \times 10^{-8} \mathrm{~m}$

$\triangle R=5.5 \times 10^{-6} \mathrm{~m}, r=1.0 \times 10^{-7} \mathrm{~m}$

- $R=1.1 \times 10^{-5} \mathrm{~m}, r=2.5 \times 10^{-8} \mathrm{~m}$

- $R=1.1 \times 10^{-5} \mathrm{~m}, r=5.0 \times 10^{-8} \mathrm{~m}$

- $R=1.1 \times 10^{-5} \mathrm{~m}, r=1.0 \times 10^{-7} \mathrm{~m}$

- $R=2.2 \times 10^{-5} \mathrm{~m}, r=2.5 \times 10^{-8} \mathrm{~m}$

- $R=2.2 \times 10^{-5} \mathrm{~m}, r=5.0 \times 10^{-8} \mathrm{~m}$

- $R=2.2 \times 10^{-5} \mathrm{~m}, r=1.0 \times 10^{-7} \mathrm{~m}$
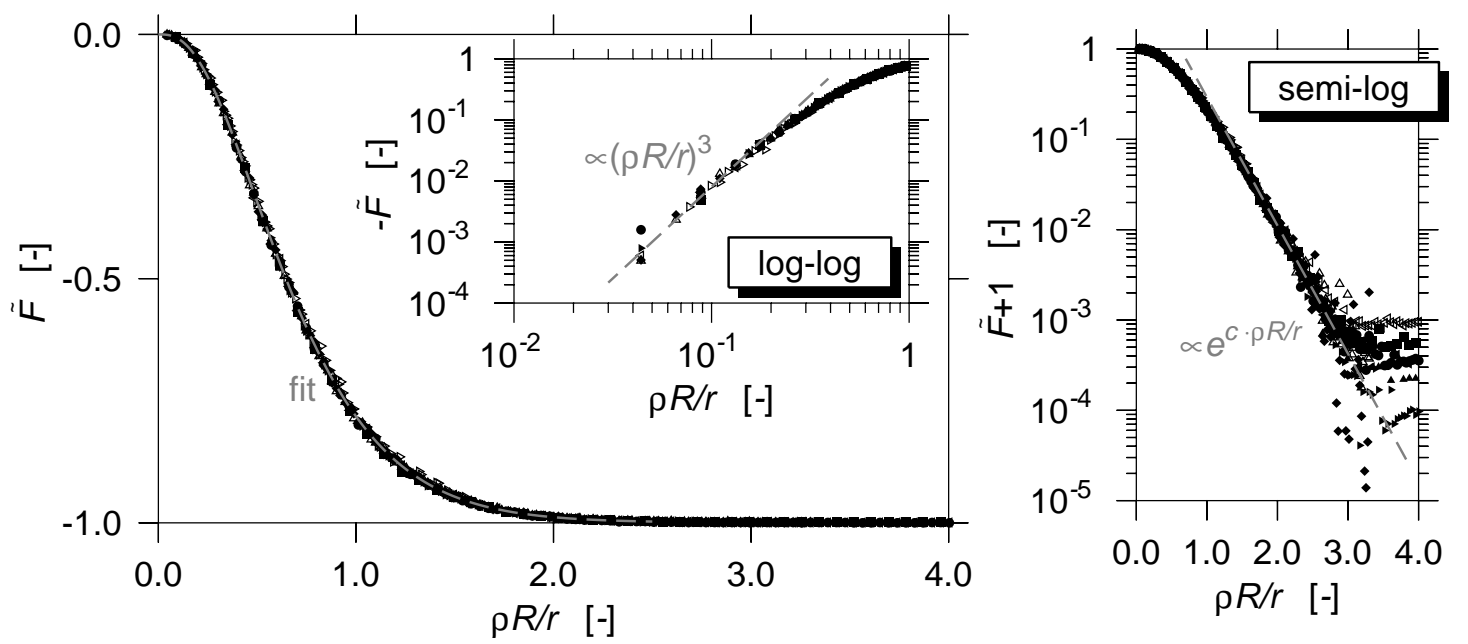

Fig. 4. (top) Semi-logarithmic plot of the rescaled simulated data $\left(A=8 \times 10^{-19} \mathrm{~J}\right.$, mean of $10^{4}$ samples per point). Increasing $\rho R / r$ the curves saturate at a constant which scales approximately as $\propto r / R$. The plot indicates a general behavior for $\rho R / r \rightarrow 0$. (bottom) The converted quantity $\tilde{F}=\frac{F-F_{0}}{F_{0}-F_{m}}$ of the same data produces a convincing data collapse. The inset and the right-hand plot indicate a power law behavior for very small $\rho R / r$ and an exponential decay shortly before the minimum is reached, respectively. The scaling function is fitted by $-0.499692 \cdot(2-\Gamma(3,4.144 \rho R / r))$.

Our previous thoughts call for an improved ansatz of the form

$$
\tilde{F}=-\frac{F_{0}(R)-F(\rho, R, r)}{F_{0}(R)-F_{m}(r)}=g\left(\frac{\rho R}{r}\right)
$$

where $g$ is a scaling function in the range $0 \leq \rho R / r \lesssim 2.5$. Here the difference of the offset $F_{0}$ to the data $F$ is determined and divided by the possible maximum of this difference, given by $F_{0}-F_{m}$. Scaling this way would give positive values with a maximum of 1 . In order to obtain a function whose 
curve represents the decrease of the adhesive force with increasing $\rho$ in a more intuitive manner, the minus sign is inserted. Now, as can be seen in Fig. 4 (bottom), we obtain a perfect collapse of the data. Note that $\tilde{F}$ does not depend on $A$, i.e., it is independent of the used materials.

\subsubsection{Asymptotic properties of the scaling function}

For $\rho R / r \rightarrow 0$ we expect a decrease of $\tilde{F}$ which is $\propto \rho^{3}$, as can be explained as follows. In the limit of low densities, $\rho \rightarrow 0$, a decrease of the adhesive force can only occur if three potential contact adsorbates lie within a critical area between the two approaching host spheres. Assuming the positions of the adsorbate particles to be uncorrelated, the probability of this event grows $\propto \rho^{3}$. A logarithmic plot verifies this power law behavior, cf. the inset of Fig. 4 (bottom).

Increasing $\rho R / r$ the semi-logarithmic plot in the right panel of Fig. 4 indicates a usual exponential decay until the minimum in $\tilde{F}$ is reached at $\rho R / r \approx 2.5$.

\subsubsection{Fit of the scaling function}

In our simulations the nanoscaled adsorbates are distributed randomly on the spherical host particle, i.e., they satisfy Poisson distributions in two dimensions, projected onto a spherical geometry. In the analysis of 2D Poisson Voronoi cells it has been demonstrated that the distribution of cell areas can be fitted to gamma distribution functions with one, two or three parameters $[8,9,10,11]$. This suggests an ansatz of the form

$$
g^{\prime}\left(\frac{\rho R}{r}\right)=\alpha\left(\frac{\rho R}{r}\right)^{\beta} \exp \left(-\gamma \frac{\rho R}{r}\right)
$$

for the derivative of the scaling function $g$, where $\alpha, \beta$ and $\gamma$ are constants. Integration yields

$$
\begin{aligned}
g\left(\frac{\rho R}{r}\right) & =\int_{0}^{\frac{\rho R}{r}} \alpha x^{\beta} \exp (-\gamma x) \mathrm{d} x \\
& =\alpha \gamma^{-\beta-1}\left(\Gamma(\beta+1)-\Gamma\left(\beta+1, \gamma \frac{\rho R}{r}\right)\right)
\end{aligned}
$$

Here $\Gamma(z)$ is the (complete) gamma function, and $\Gamma(a, z)$ is the (upper) incomplete gamma function. 


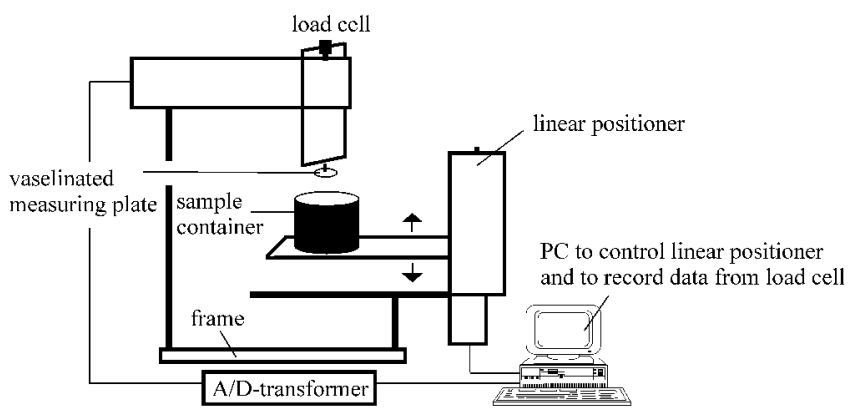

Fig. 5. Scheme of the tensile tester.

Since $\tilde{F}$ decreases as $\rho^{3}$ if $\rho$ is small (see above), we expect the exponent $\beta=2$. Indeed fitting Eq. (8) to our data in the range $0 \leq \rho R / r \leq 2.5$, one obtains $\beta=2.00 \pm 0.01$. Setting $\beta=2$ and performing another fit, we determined the fit parameters as $\alpha=-35.56 \pm 0.07$ and $\gamma=4.144 \pm 0.003$. So the numerical result for the scaling function is

$$
g\left(\frac{\rho R}{r}\right)=-0.500 \cdot\left(2-\Gamma\left(3,4.14 \frac{\rho R}{r}\right)\right) .
$$

In Fig. 4 (bottom) one can see that it matches the rescaled numerical data perfectly.

\subsection{Comparison to experiments}

The theoretical results can be compared to previous experiments with a tensile tester [2], cf. Fig. 5 .

In this experiments a vaselinated measuring plate is brought into contact with the surface of a powder sample. Lifting the plate a tensile force is measured which allows one to determine the interparticle forces needed to separate two powder layers (for details see Appendix C).

A comparison of the experimental data with the results of the simulation is shown in Fig. 6. Here the quantity of the simulated adhesive force $F$ is arbitrarily scaled against the quantity of the experimentally measured tensile strength $\sigma$ so that the offsets collapse. For $\rho=0$ there are no nanoscaled adsorbates on the host particle, and so $F$ and $\sigma$ are generated only by powder-powder interactions. Although the experimentally measured forces for $\rho>0$ are systematically larger than the predicted ones, they show a qualitatively similar type of curvature, confirming the approach of studying random coverages.

At present the origin of the systematic deviations is not yet entirely clear. 

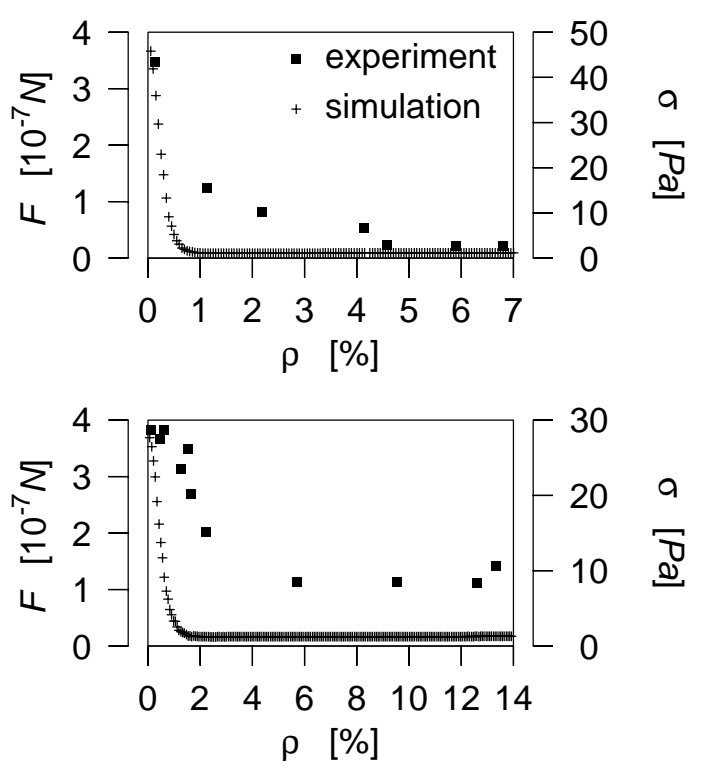

Fig. 6. Qualitative comparison of the simulated adhesive force $F$ with experiments in which the tensile strength $\sigma$ was measured; note that these are two different physical quantities $F$ and $\sigma$, scaled against each other arbitrarily in order to give combinations showing common trends in simulation and experiment. The experimental data is taken from [12]. (top) After some mixing, by which agglomerates of the nanomaterial are crushed into smaller aggregates, aggregates of Aerosil ${ }^{\circledR} 300$ deposited on corn starch powder particles $(R=11 \mu \mathrm{m})$ have average radii of $r=42 \mathrm{~nm}$. The simulation used $A=6.5 \times 10^{-20} \mathrm{~J}$ as Hamaker constant for this material combination. (bottom) Analogous graph for Aerosil ${ }^{\circledR}$ OX 50 on corn starch with $R=11 \mu \mathrm{m}$, $r=80 \mathrm{~nm}$ and $A=6.5 \times 10^{-20} \mathrm{~J}$.

They could be attributed to the influence of additional liquid bridges between the powder particles. However, with corn starch the formation of liquid bridges is only observed at relative humidities of more than $40 \%$ [12], and all experimental measurements were performed well below this critical limit.

Aggregation is another important aspect. The adsorbate particles mentioned above are aggregates of nanoscaled primary particles [2]. Depending on the number of combined primary particles the diameters of the adsorbates vary. The radii $r$ used in our simulations are averages over many adsorbates whose sizes were measured by atomic force microscopy during the experiments. Experimentally the measured radii have errors of the order of about $r / 2$ [12]. However, in the simulation statistical fluctuations of the adsorbate radii are not included in order to keep the problem simple and fast computable. In this way we make systematic errors, but a relative error in $r$ of about 0.5 alone cannot explain the quantity of the deviations between simulation and experiment, cf. Fig. 6.

The precise three-dimensional shapes of the adsorbates are also not yet completely analyzed. Experimentally the aggregates are placed onto the surfaces 
of the host particles by simply mixing the nanomaterial with the powder. The number of adsorbed aggregates increases during mixing, and the surface coverage of the host particles grows. As an unintended side effect of this mixing a 'grinding' of the aggregates occurs [1]. While at the beginning of the mixing process we expect the shape of the adsorbed aggregates to be spheric as an approximation, their shape gets continuously flatter due to the mechanical stress during mixing. So we expect a deviation upwards from the computed adhesive force because flat adsorbates generate a smaller distance between two powder particles than the spherical adsorbates assumed in the simulation.

Additionally, it seems to be likely that the experimental method overestimates the actual forces. In fact, the top layer separated by the tensile tester is not a monolayer of spheres. Instead we expect a rough and irregular surface, especially at the edges of the sample, and so the force needed to produce such a surface is presumably higher.

Nevertheless the systematic nature of the deviations allows us to conclude that the simulation results may serve as lower bounds to the actual forces and to the minimum surface coverage needed to optimize the powder flow.

\section{Conclusions}

In the present work we have investigated how van der Waals forces between two spherical particles are reduced by randomly deposited nanoscaled adsorbates. In contrast to an earlier work by Meyer, who considered a triangular coverage, the statistical analysis of randomly deposited nanoadsorbates leads to an improved prediction of the force reduction as a function of the coverage. More specifically, a continuous curve instead of a step-like function is obtained. Varying parameters we have identified scaling laws which are independent of the used materials. A comparison with previously recorded experimental data shows that the force predicted by the simulation may be used as a lower bound for the actual force, though the experimental data is not sufficient and not precise enough to verify the functional behavior properly. This could be a starting point for further experiments. Future simulations could include a statistical distribution of the adsorbate size. However, the decline of the adhesive force computed in our model may act as a lower approximation of the minimum surface coverage which is needed to optimize the powder flow. 


\section{A Simulation in detail}

\section{A.1 Preparation}

For small $\rho$ there is no difference - respecting the problem - whether to regard two covered powder particles, or one uncovered particle and one which is covered at double density, cf. Fig. 1. Thus, for simplicity, the preparation part of the simulation covers only one host particle stochastically with the coverage density $2 \rho$.

The center of the sphere to be covered is the origin in our coordinate system. Random sphere point picking must be done with respect to the sphere's surface area. In terms of spherical coordinates, where $\theta$ is the polar and $\varphi$ the azimuthal angle,

$$
\mathrm{d} \Omega=\mathrm{d} \varphi \mathrm{d} \theta \sin \theta=-\mathrm{d} \varphi \mathrm{d}(\cos \theta)
$$

is an area element on the unit sphere. So a uniform point distribution is achieved by rectangular distributions of

$$
\varphi \in[0,2 \pi) \text { and } \cos \theta \in[-1,1]
$$

As a further simplification, we cover only one half of the first sphere, where the contact to the second, uncovered sphere will be made. The neglected adsorbates would hardly contribute to the bonding force, as they would be too far away from the second powder particle.

In this way adsorbates are successively placed upon the host, with their centers at coordinates $\theta_{i}, \varphi_{i}$, until the desired coverage is reached. Let $O_{S}=4 \pi R^{2}$ be the host's surface area and $C_{A}=\pi r^{2}$ the area of perpendicular projection of an adsorbate on this surface. So the number of adsorbates on the host sphere can be calculated from the relative surface coverage $\rho$ as

$$
N_{A} \cong \rho \frac{O_{S}}{C_{A}}=4 \rho\left(\frac{R}{r}\right)^{2}
$$

Note that the factor 2 due to covering only one host particle and the factor $\frac{1}{2}$ due to covering only half this particle cancel each other out. 


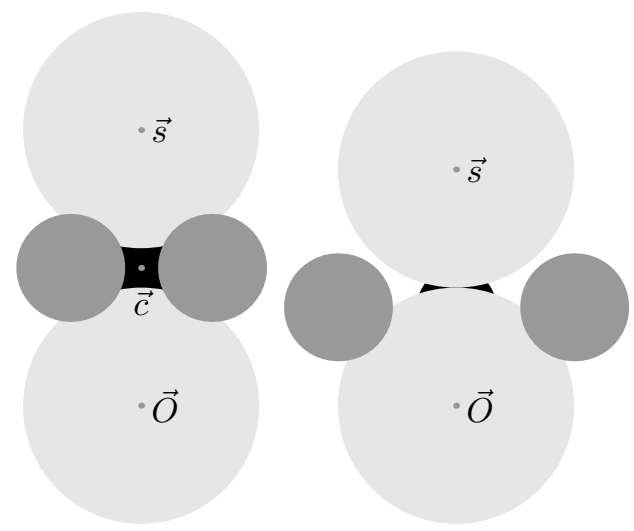

Fig. A.1. (left) If the circumcenter $\vec{c}$ of the contact adsorbates is more than $D / 2$ away from the surface of the covered powder particle (located at the origin $\vec{O}$ ), the second powder particle is placed in such a way that $\vec{c}$ bisects the distance between these two powder particles. (right) If $\vec{c}$ is less than $D / 2$ away from the surface of the covered powder particle, i.e., that the two powder particles will touch each other, the second powder particle is placed in the direction of $\vec{c}$ so that there is just the contact distance $D$ between the two powder particles.

\section{A.2 Positioning}

After complete preparation of the first sphere, the second uncovered sphere approaches along the $z$-axis in negative direction, until it hits the covered hemisphere. In order to determine the three contact points as easy as possible, the coordinates of the $N_{A}$ adsorbates are transformed by projection on the $x$ $y$-plane, $(x, y, z) \rightarrow(x, y)$, which is a sufficient approximation for $r \ll R$.

The roll-off mechanism described in Fig. 2 and its caption yields the three contact points; consequently the contact position of the second powder particle can be calculated, which will be explained in the following. With the coordinates of the centers of the three contact adsorbates the circumcenter $\vec{c}$ of the triangle spanned by these three points can easily be constructed. Note that the center of the covered powder particle was defined to be located at the origin. So for symmetry reasons, see Fig. A.1, the center position $\vec{s}$ of the second, uncovered particle can be computed as

$$
\vec{s}=\left\{\begin{array}{ll}
2 \vec{c} & \text { if } \quad|\vec{c}| \geq R+\frac{D}{2} \\
(2 R+D) \frac{1}{|\vec{c}|} \vec{c} & \text { if } \quad|\vec{c}|<R+\frac{D}{2}
\end{array} .\right.
$$

As the algorithm for finding the three-point contact does not check for a direct contact between the two host particles, the artificial definition of $\vec{s}$ for $|\vec{c}|<$ $R+\frac{D}{2}$ must be introduced. So we ensure a minimum distance of $D$ between the two host particles. For this case of contacted powder particles the adsorbates 


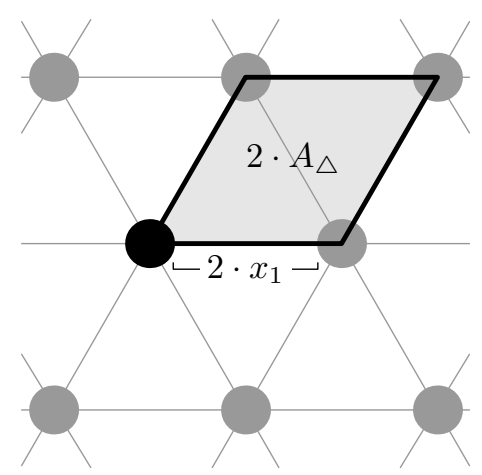

Fig. B.1. Surface layout according to Meyer's model. The nanoadsorbates (large dots) occupy the vertices of the equilaterally triangulate surface of the powder particle (white background). Distributing the surface area equally to the adsorbates, the area of the highlighted cell belongs to each individual one.

are of minor importance, since the direct powder-powder interaction exceeds any powder-adsorbate interaction by some orders of magnitude.

\section{B Conversion of the parameters from Meyer's model}

In order to be able to compare Meyer's model with our simulated data, one has to convert the control parameter used in this study, the relative surface coverage $\rho$, into the quantity $x_{1}$ used by Meyer, cf. Fig. B.1 and [3,2].

Eq. (A.3) yields the total number of adsorbates on one host particle, $\rho \mapsto N_{A}$. Although it is not possible to cover the surface of a sphere globally by a hexagonal lattice it is assumed that such a structure can be established locally. According to Meyer's model, adsorbates are set on the vertices of this lattice, so that a uniform pattern of equilateral triangles evolves. To each adsorbate belongs a cell of the area

$$
2 \cdot A_{\triangle}=\frac{4 \pi R^{2}}{N_{A}}
$$

cf. Fig. B.1. With elementary geometrical considerations one can calculate the side length of an equilateral triangle of the area $A_{\triangle}$ as

$$
a=\frac{2}{\sqrt[4]{3}} \cdot \sqrt{A_{\triangle}}
$$

Consequently

$$
x_{1}=\frac{a-2 r}{2}=\frac{a}{2}-r
$$




\begin{tabular}{|l|c|c|c|}
\hline Nanomaterial & $\begin{array}{c}\text { Diameter of the primary particles } \\
{[\mathrm{nm}]}\end{array}$ & $\begin{array}{c}\text { Solid density } \\
{\left[\mathrm{g} / \mathrm{cm}^{3}\right]}\end{array}$ & $\begin{array}{c}\text { Specific surface } \\
{\left[\mathrm{m}^{2} / \mathrm{g}\right]}\end{array}$ \\
\hline Aerosil $^{\circledR} 300$ & 7 & 2.2 & $300 \pm 30$ \\
\hline Aerosil $^{\circledR}$ OX 50 & 40 & 2.2 & $50 \pm 15$ \\
\hline
\end{tabular}

Table C.1

Specific properties of the used nanomaterials.

follows, which can be put in the formulas for $h\left(x_{1}\right)$ and $F_{\mathrm{vdW}}(h)$, see Eqs. (27) and (24) in [3]. Hereby a mapping $\rho \mapsto F_{\mathrm{vdW}}$ gets feasible.

\section{Experiments}

\section{C.1 Materials and methods}

Corn starch, Cerestar ${ }^{\circledR}$ GL 03406, was purchased from Cerestar Germany, Krefeld. The spherical particles have diameters of $22 \mu \mathrm{m}$. They are very cohesive. As nanomaterials we used Aerosil ${ }^{\circledR} 300$ and Aerosil ${ }^{\circledR}$ OX 50 both supplied by DEGUSSA AG, Hanau, Germany. Their specific properties are summarized in Table C.1.

Both types of Aerosil ${ }^{\circledR}$ are hydrophilic. Due to their small sizes they highly agglomerate.

In our experiments we determined the tensile strengths of powder mixtures consisting of corn starch and $0.2 \%$ of a nanomaterial. In order to prepare these mixtures first the nanomaterial was given into a glass vial with a volume of $500 \mathrm{ml}$. Then in a second step $100 \mathrm{~g}$ of corn starch were added. The degree of filling was given by 0.4 . The powders were mixed by means of a Turbula mixer (Type T2C, Nr. 950353, W. Bachofen AG, Basel, Switzerland) at $42 \mathrm{rpm}$. A comparison of the particle sizes of the mixture components suggests that the mixing process involves a grinding of the agglomerates formed by the nanomaterials.

\section{C.2 Measurement of tensile strengths}

The interparticle forces acting in the mixtures consisting of corn starch and $0.2 \%$ of a nanomaterial were measured by means of tensile strength tester developed by Schweiger [13,14] and modified by Anstett [15], Fig. 5.

The powder to be tested is filled into the sample container having a volume 
of $4.62 \mathrm{~cm}^{3}$ (diameter: $3.43 \mathrm{~cm}$, height: $0.50 \mathrm{~cm}$ ). By means of a sieve with a sieve size of $315 \mu \mathrm{m}$ it is sieved directly into the sample container. In order to achieve a homogenous filling the filled container is gently tapped onto a soft pad. By scraping off the surplus testing material by means of a slide a perfectly flat surface of the powder bed can be obtained. This step has to be performed very carefully in order to avoid even the slightest compression of the powder.

The sample container prepared in this way is placed on a small table which can be moved up and down by means of the linear positioner M-410-21 (Physik Instrumente GmbH\&Co, Waldhorn). By coarse adjustment screws the whole unit consisting of a table as well as a linear positioner is moved upwards so that only a very small gap of about 1 to $2 \mathrm{~mm}$ remains between the powder surface and the measuring plate. From this position on the further movement of the table by the linear positioner is controlled by the controller C-832.00 (Physik Instrumente GmbH\&Co, Waldhorn) which runs under the software package Pro Move v2.20. The steps performed by the linear positioner can be simultaneously seen on the screen of the PC. With a vertical speed of $3.4 \mu \mathrm{m} / \mathrm{s}$ the table is moved upwards until the measuring plate comes into contact with the powder surface. The movement is stopped as soon as a defined negative weight of the measuring plate is measured.

The cylindrical measuring plate has a lower surface of $0.385 \mathrm{~cm}^{2}$. By means of a frame made from Aluminum it is fixed on a load cell ZER 10 (Wipotec GmbH, Kaiserlautern, Germany). The measuring range of this load cell covers a range of $2 \mathrm{~g}$ with a resolution of $0.1 \mathrm{mg}$. The force acting on the load cell is registered by the software Wipotec-Terminal v3.78 at a scan rate of three measurements per second. The signals of the load cell are mass calibrated. In order to obtain the corresponding forces the signals have to be multiplied by the gravitational constant $g$.

Before each measurement a solution of $5 \%(w / w)$ white petrolatum in petrolether is sprayed onto the lower side of the measuring plate. After evaporation of the solvent the measuring plate is coated with a thin layer of petrolatum as a sticking agent to ensure that the powder particles adhere on its surface.

After a pause of $10 \mathrm{~s}$ the table with the sample container is moved downwards at a speed of $1.7 \mathrm{~m} / \mathrm{s}$. Due to the interaction between the layer of powder particles fixed at the lower side of the measuring plate and the particles remaining in the adjacent powder layer in the sample container the load cell measures a force. This force increases with continuing downwards movement of the sample. It reaches its maximum when the two powder layers separate. The force measured by the load cell drops to that force corresponding to the weight of the powder layer adhering at the lower surface of the measuring plate, Fig. C.1. 


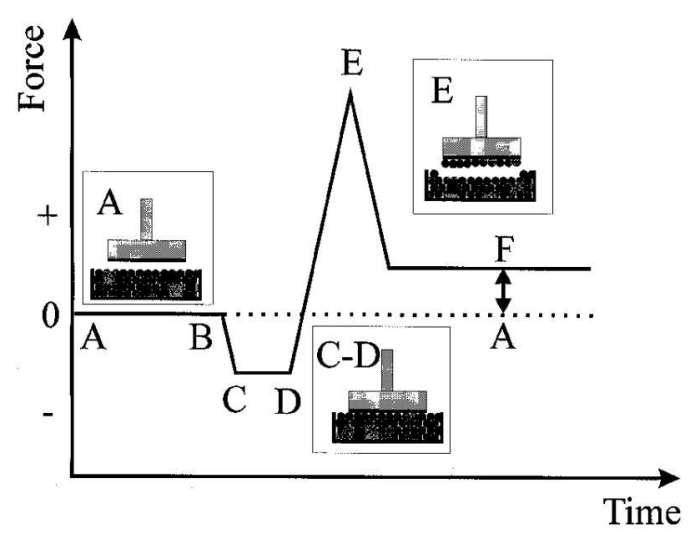

Fig. C.1. Time course of the force during a measuring cycle.

In order to calculate the tensile strength $\sigma$ the force $F_{F}$ measured at point $\mathrm{F}$ is subtracted from the maximum force $F_{E}$ measured at point E. This value is then divided by the lower surface $A_{M}$ of the measuring plate:

$$
\sigma=\frac{F_{E}-F_{F}}{A_{M}}
$$

\section{References}

[1] K. Meyer, I. Zimmermann, Effect of glidants in binary powder mixtures, Powder Technology 139 (2004) 40-54.

[2] K.

Meyer, Nanomaterialien als Fließregulierungsmittel, Dissertation, Universität Würzburg (2003).

(http://opus.bibliothek.uni-wuerzburg.de/opus/volltexte/2003/559/)

[3] I. Zimmermann, M. Eber, K. Meyer, Nanomaterials as flow regulators in dry powders, Zeitschrift für Physikalische Chemie 218 (2004) 51-102.

[4] H.C. Hamaker, The London-van der Waals attraction between spherical particles, Physica IV 10 (1937) 1058-1072.

[5] H. Rumpf, Die Wissenschaft des Agglomerierens, Chemie Ingenieur Technik 46 (1974) 1-11.

[6] J.N. Israelachvili, Intermolecular and surface forces, Academic Press (1985) London, Second Printing 1987.

[7] H. Schubert, Food particle technology. Part I: Properties of particles and particulate food systems, Journal of Food Engineering 6 (1987) 1-32.

[8] D. Weaire, J.P. Kermode, J. Wejchert, On the distribution of cell areas in a Voronoi network, Philosophical Magazine B 53 (1986) L101-L105. 
[9] S. Kumar, S.K. Kurtz, Properties of a 2-dimensional Poisson-Voronoi tesselation - a Monte-Carlo study, Materials Characterization 31 (1993) 5568 .

[10] A.L. Hinde, R.E. Miles, Monte-Carlo estimates of the distributions of the random polygons of the Voronoi tessellation with respect to a Poisson-process, Journal of Statistical Computation and Simulation 10 (1980) 205-223.

[11] K. Marthinsen, Comparative analysis of the size distributions of linear, planar, and spatial Poisson Voronoi cells, Materials Characterization 36 (1996) 53-63.

[12] M. Eber, Wirksamkeit und Leistungsfähigkeit von nanoskaligen Fließregulierungsmitteln, Dissertation, Universität Würzburg (2004). (http://opus.bibliothek.uni-wuerzburg.de/opus/volltexte/2004/902/)

[13] A. Schweiger, Untersuchungen zum Fließverhalten feinkörniger Schüttgüter, Dissertation, Universität Würzburg (1998).

[14] A. Schweiger, I. Zimmermann, A new approach for the measurement of the tensile strength of powders, Powder Technology 101 (1999) 7-15.

[15] I. Anstett, Untersuchungen zur Wirkungsweise von Fließregulierungsmitteln auf partikulärer Ebene, Dissertation, Universität Würzburg (2001). 